\title{
Posturodynamic 6 Test: A New Scoring Method for Effective Communication of Results
}

\author{
Alice Janin'1, José Angelo Barela², Philippe Dupui'3, Michael T. Gross', Marc Janin ${ }^{5}$ \\ ${ }^{1} 7$ Rue de Tréguel 86000 Poitiers, France \\ ${ }^{2}$ Institute of Physical Activity and Sport Sciences, Cruzeiro do Sul University, São Paulo, Brazil \\ ${ }^{3}$ Laboratoirede Physiologie, Faculté de Médecine de Rangueil, Toulouse, France \\ ${ }^{4}$ FAPTA Program in Human Movement Science, Division of Physical Therapy, University of North Carolina at \\ Chapel Hill, Chapel Hill, USA \\ ${ }^{5}$ Applied Podiatry College, Poitiers, France \\ Email: jose.barela@cruzeirodosul.edu.br, philippe.dupui@univ-tlse3.fr,mike_gross@med.unc.edu
}

Received 8 December 2015; accepted 26 January 2016; published 29 January 2016

Copyright (C) 2016 by authors and Scientific Research Publishing Inc.

This work is licensed under the Creative Commons Attribution International License (CC BY). http://creativecommons.org/licenses/by/4.0/

(c) (i) Open Access

\section{Abstract}

Background: Posturodynamic 6 (PDN-6) is a clinical assessment of posture that merges the Clinical Posturodynamic Test and the Pelvic Maintain Test. Current scoring system does not fulfill all our needs and requirements mostly because the same numeric score might reflect 28 different possible combinations of postural dysfunction in terms of anatomic region and laterality. Objective: We propose a new scoring method for the PDN-6 that would not change the clinical methods for the PDN-6 assessment. Specifically, new scoring method would clearly indicate specific patterns of postural dysfunction while still enabling statistical analyses. Methods: We developed a new scoring method for the PDN-6 without changing the instrument's clinical procedures. We qualitatively assessed the validity of the new scoring system to detail specific patterns of postural dysfunction in terms of anatomic region and laterality. Results: New scoring method successfully deals with limitations of the previous scoring method. The new method enables clinicians to differentiate among 2 or more patients who might have very different patterns of postural dysfunction while still having the same numeric score using the previous scoring. The new scoring method provides quantitative data that are easily translated in terms of anatomic region and laterality for the postural dysfunctions that are present. Patient behavioral improvements are quantified, documented and interpreted with a change in score, and the exact nature of the improvements can be determined in terms of anatomic location and laterality. Rash analyses can also be used for statistical analyses. Conclusion: PDN-6 new scoring method provides quantitative data that provide more specific information about a patient's postural deficits and any changes in their postural dysfunction over time without changing the clinical assessment methods. 


\section{Keywords}

\section{Posture, Clinical Evaluation, Spine, Balance, Balance Function, PDN}

\section{Introduction}

The Posturodynamic 6 (PDN-6) is a novel method of rating posture using 6 assessment items and a simple measurement scale. The purpose of the PDN-6 is to assist in the evaluation of standing posture, but not to replace other more involved clinical assessments of standing posture. The PDN-6 represents a combined modification of two clinical instruments for postural evaluation: the Clinical Posturodynamic Test (CPT) and the Pelvic Maintain Test (PMT). The traditional method for scoring the PDN-6 has several limitations in terms of not being able to identify the anatomic region of dysfunction and the inability to communicate areas of improvement or exacerbation over time. The purpose of our report is to describe these limitations in detail. We also propose a new scoring system for this instrument that will enable the specific identification of postural deficits and will communicate clearly any positive or negative changes in postural function.

\section{The CPT and PMT Instruments}

The CPT is a clinical test with 4 evaluation items (cervical, thoracic, lumbar, and pelvis) for the right and for the left sides of the body [1]. This instrument assesses the postural response of each spinal section and pelvis during a lateral flexion movement [1]-[3]. The practitioner observes the result of the physiological response of the spine range of motion [4]. The quality of the postural response is quantified through spinal lateral flexion and pelvic translational maneuvers. The practitioner positions their hands successively on the four anatomical regions that are evaluated: cervical spine, thoracic spine, lumbar spine, and pelvic girdle. For the first three anatomic regions, the patient is instructed to laterally flex in the frontal plane: "slowly slide your hand down the side of your leg to the left and then to the right”. The normal physiologic response of the lower thoracic and lumbar regions is characterized by a slowly progressive contra-lateral rotation. Ipsilateral rotation should occur in the cervical region and upper thoracic regions of the spine because of the orientation of the articular facets. When the practitioner induces a lateral movement of the pelvic region to the right or left, the normal physiological response is a slow but progressive contra-lateral rotation (i.e. if the lateral movement induces by practitioner is on the right, the physiological response is pelvic rotation on the left) [1] [2] [4]. Previous research indicates that the CPT, in general, has $80 \%$ intra-examiner reliability and that intra-examiner reliability varies depending on the anatomic location [2] [3]. In clinical practice, however, we recommend that CPT measurements be made by the same clinician to improve the reliability of repeated measurements. Observations made during the CPT assessment are reported on a clinical data sheet (Figure 1(a)). If the response of the anatomic region is not a normal physiologic response, the practitioner records an " $\mathrm{X}$ " on the clinical data sheet and nothing if the patient's response is a normal physiological response [1] [2]. With 2 possible observations for each of the 8 assessments, 30 possible combinations of overall performance on the CPT are possible. The CPT is scored numerically by replacing every " $X$ " with the number " 1 " and then adding all of the recorded 1's for a total score on the CPT. In the examples given in Figure 1, the CPT score is 0 for Figure 1(a) and the score is 6 for the CPT depicted in Figure 1(b).

The PMT combines the Trendelenburg's Sign (TS) [5] and the Single-Limb Stance (SLS) test [6]. The TS and the SLS test are timed measures that assess postural steadiness as the patient holds a static position. The popular belief is that better postural steadiness is reflected by longer standing time on a single leg. Little evidence is available, however, regarding how postural steadiness during one-legstance changes over time. The TS and SLS tests evaluate the patient's response during singleleg standing. The clinician stands behind the patient for the PMT and places his hands on the patient's iliac crests and his thumb's on the patient's posterior superior iliac spines. The subject stands on one lower extremity, slightly bending the knee of the non-stance limb. The normal response for the TS test should be that the patient's trunk remains erect and the contralateral iliac crest should either remain level with the iliac crest of the stance limb, or should elevate slightly higher than the stance limb's iliac crest. If the non-stance limb's iliac crest falls inferior to the level of the stance limb's iliac crest, then the 


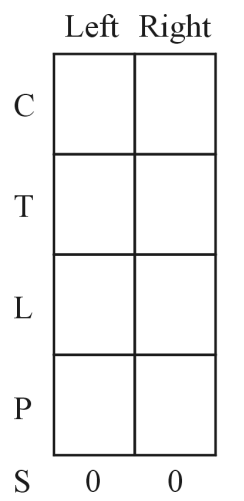

(C: cervical; T: thoracic; L: lumbar; P: pelvis; S: score)

(a)

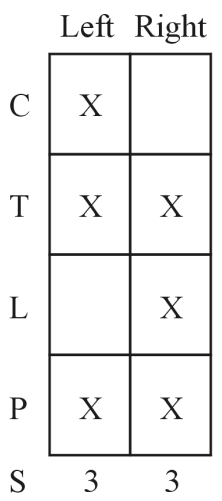

(X: no physiological responses; C: cervical; T: thoracic; L: lumbar; P: pelvis; S: score)

(b)

Figure 1. Datasheet for the clinical posturodynamic test.

examiner records a positive TS test result. A positive test result suggests reduced strength or activation of the hip abductor muscles of the stance limb [6]-[9]. A positive test result on the TS test is recorded as an " $\mathrm{X}$ " on the clinical data sheet.

The normal physiologic response for the 30 second SLS test involves the patient's spine being maintained in a vertical position, while the iliac crests remain level with no compensatory movements made by the non-weight bearing lower extremity or the upper extremities for the duration of the test. Any deviation from these normal positions is recorded as an " $\mathrm{X}$ " on clinical data sheet and the time from the beginning of the test to the postural fault is recorded in seconds [10] [11]. The test is conducted and responses are recorded for both right and left stance limbs (Figure 2).

The combined TS and SLS scores for the PMT allow the clinician to identify stability problems that may exist for issues such as proprioceptive dysfunction. The PMT is positive (abnormal response) when the patient cannot maintain a level pelvis for 30 seconds without compensatory balancing movements by the upper extremities or the contra-lateral lower extremity. An abnormal response for the PMT is recorded on the data sheet with an "X" if the patient cannot maintain a level pelvis for 30 seconds, and the clinician indicates the elapsed time in seconds that the patient was able to maintain normal alignment until the postural fault occurred (Figure 2).

As previously stated, the PDN-6 represents the union of the CPT and PMT instruments. The PDN-6 datasheet reflects a scoring method for the 2 tests combined (Figure 3(a)) and provides both quantitative and qualitative information. The qualitative information reflects the postural responses of the patient during movement and static positioning tasks. Abnormal responses are recorded as an " $X$ " on the data sheet (Figure 3(b)). The quantitative information is the time in seconds (up to a maximum of 30 seconds) that the patient was able to maintain normal pelvic position in the frontal plane during the static standing tests (Figure 3(a), Figure 3(b)). The score of each of the two example datasheets represented in Figure 3 is 6 .

\section{Limitations of the Current Scoring System for the PDN-6}

All of this information leads to a discussion of the benefits and limitations of the scoring method that has just been described. One benefit of this scoring method is that the quantitative scores derived from the PDN-6 are available for statistical analysis procedures. An examination of the sample data sheets in Figure 3, however, reveals the limitations of this scoring method. Both data sheets in Figure 3 have the same quantitative score of 6, but a close examination of the data sheets indicates that the 2 patients have very different anatomical regions of dysfunction. The quantitative scoring method, therefore, does not have the ability to discriminate different dysfunction profiles. Statistical analysis, therefore, may not be able to discriminate dysfunction among patients, despite the presence of real clinical differences. Extreme scores may be able to differentiate between widespread postural dysfunction and normal postural responses, but the scoring system may not be sensitive to intermediate scores and nominal variations between individuals. We propose, therefore, a new scoring system for the PDN-6 to address these limitations. 


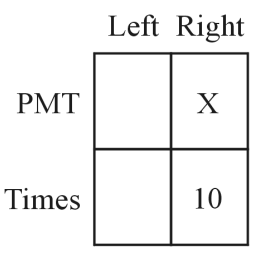

(X: no physiological responses; PMT: Pelvic Maintain Test;

10: only ten seconds maintained on one leg stance)

Figure 2. Datasheet for the pelvic maintain test (PMT).

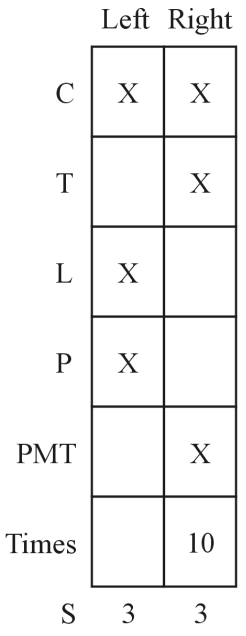

(X: no physiological responses; C: cervical; T: thoracic; L: lumbar; P: pelvis; S: score; PMT: Pelvic Maintain Test; $\mathrm{S}$ : score; 10: only ten seconds maintained on one leg stance)

(a)

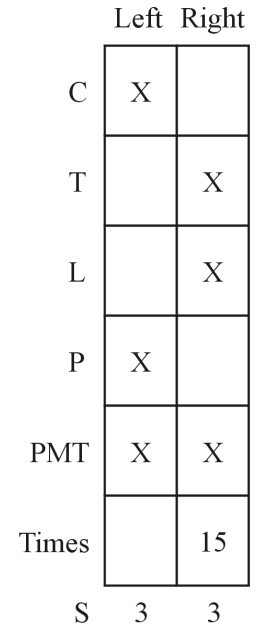

(X: no physiological responses; C: cervical; T: thoracic; L: lumbar; P: pelvis; S: score; PMT: Pelvic Maintain Test; $\mathrm{S}$ : score; 15: only fifteen seconds maintained on one leg stance)

(b)

Figure 3. Datasheet for the posturodynamic 6 (PDN-6).

\section{Proposed New Scoring System for the PDN-6}

We propose a new scoring strategy for the PDN-6 to address the previously described limitations. In doing so, we hope to retain the quantitative and qualitative information and still have a scoring method that will enable valid statistical analysis methods. Therefore, we propose a new scoring method without changing the clinical testing procedures and the data sheet format. The new scoring method simply involves numerical score transformations of the observed behavioural responses by the patient.

For the original scoring method, an abnormal physiologic response was always recorded as an " $\mathrm{X}$ " and then transformed to a numerical " 1 " for the purpose of adding a total score for the test. We now propose that abnormal responses be scored as: cervical $=1$, thoracic $=2$, lumbar $=4$, pelvic $=10$, and PMT $=100$. The clinician would still record the time that the patient was able to maintain a level pelvis during the PMT test if the patient could not maintain a level pelvis for 30 seconds.

This method of scoring allows some differentiation among the various anatomic segments with the quantitative score. We also propose that separate total scores should be computed for the right and left sides, and that these scores be registered at the bottom of the data sheet and separated by a comma to assist in describing the laterality of any dysfunctions that exist. Sample scores are provided using this method in Figure 4, Figure 5(a), and Figure 5(b).

The sample data sheet in Figure 5(b) demonstrates how the new quantitative scoring method also provides qualitative information regarding the anatomic locations where postural dysfunction has been detected. A numerical score of " 5 " to the left of the comma can immediately be translated into cervical and lumbar dysfunction on the left side without examination of the data sheet. A numerical score of 112 to the right of the comma must coincide with thoracic and pelvic postural dysfunction as well as a positive PMT. This scoring method will still 


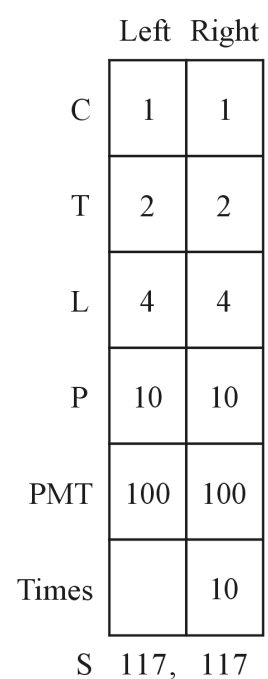

(C: cervical; T: thoracic; L: lumbar; P: pelvis;

S: score; PMT: Pelvic Maintain Test; S: score;

10: only ten seconds maintained on one leg stance)

Figure 4. New scoring in function of anatomical situation.

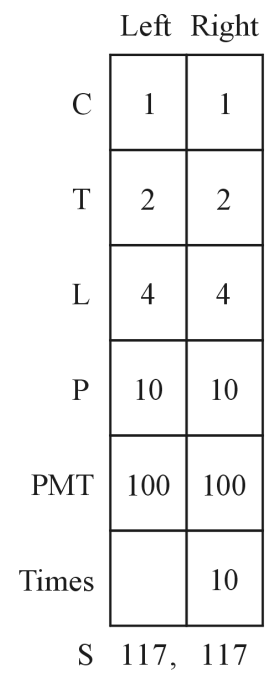

(C: cervical; T: thoracic; L: lumbar; P: pelvis; S: score; PMT: Pelvic Maintain Test; S: score; 10: only ten seconds maintained on one leg stance. Score is 117,117 )

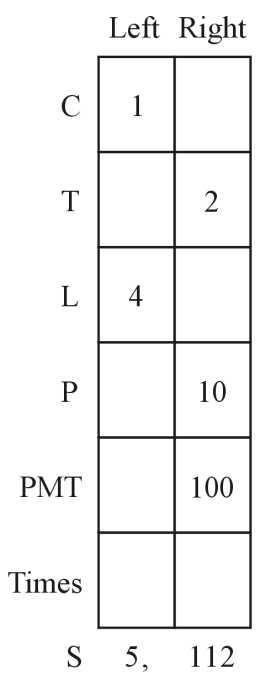

(C: cervical; T: thoracic; L: lumbar; P: pelvis; S: score; PMT: Pelvic Maintain Test; S: score; 10: only ten seconds maintained on one leg stance. Score is 5, 112)

\section{Figure 5. Variation of the new scoring in function according to the anatomical situation.}

make possible the use of non-parametric statistical analyses of the quantitative raw scores. Moreover, the quantitative scores will also make possible full communication among clinicians regarding the anatomic location and laterality of any postural dysfunctions that are present.

The previous quantitative scoring method for the PDN-6 conveyed, in general, the degree to which a patient demonstrated postural dysfunction. Greater scores suggested widespread postural dysfunction and lesser scores suggested far less postural dysfunction. The exact locations of dysfunction, however, could not be determined. Reductions in total PDN-6 scores indicated improvement, but did not identify the anatomic region or laterality of the improvement. The new scoring method successfully addresses all of these issues.

Figure 5(a), and Figure 5(b) represent pre-intervention $(117,117)$ and post-intervention $(5,112)$.

PDN-6 assessments for a hypothetical patient. These scores readily reveal the nature of the therapeutic- 
induced changes for this patient. Prior to the treatment, the numeric score of " 117,117 ” clearly indicates postural dysfunction for all segments as well as the PMT on both the left and right sides of the body. The postintervention score of " 5,112 " indicates that postural faults have been resolved on the left except for the cervical and lumbar regions, and that only the cervical and lumbar postural dysfunctions have been resolved on the patient's right side. All of this information can be determined from the numerical scores without examining the patient's data sheets.

The new scoring method that we have suggested does not change the clinical testing procedures that have been used in the past for the PDN-6 instrument. The patient should begin the test by standing in their relaxed and comfortable stance position with double limb support. The patient's arms should be resting at their side and they should be looking straight ahead. During the assessment, the patient should not turn around toward the clinician since this will significantly affect their posture. The total time to complete all PDN-6 assessment procedures should be approximately two minutes. The clinician needs to have unencumbered access to the posterior aspect of the patient during the assessment, and all procedures for the assessment should be performed by the same clinician as per previous literature recommendations regarding intra-tester reliability [12]. To our knowledge, this is the first attempt to improve the scoring of the PDN-6 by addressing the previously described limitations of the current scoring system.

\section{Limitations}

Similar to the Foot Posture Index, the PDN6 is a qualitative assessment instrument. The obtained scores will range from 0 to 117 and are not on a ratio scale. Parametric data analyses are not appropriate since the data for this scoring system are not ratio data [13]. Other analysis methods such as Rasch analyses may be more appropriate for larger PDN-6 data sets to consider how the person/item interaction is governed by the difficulty of test forms (examiners + tasks + items) and the ability of the subject to perform the task [14] [15].

\section{Conclusion}

The new scoring method for the PDN-6 enables rapid and specific translation of a numerical score that indicates both the specific anatomic location and laterality of postural dysfunctions. This capability is possible without changing any of the testing procedures for the PDN-6. The scoring method also helps clinicians document and communicate specific details regarding improvements or exacerbations of a patient's postural function, as well as enables statistical analyses of the data. In the clinical setting, however, the examination procedures for the PDN-6 using this new scoring system are standardized so that data for the PDN-6 should more accurately reflect the patient's status compared with the original scoring system.

\section{Acknowledgements}

We thank Stuart M. McGill, Professor of Spine Biomechanics, Faculty of Applied Health Sciences, University of Waterloo, Waterloo, Ontario, Canada for personal comments about this work.

$\mathrm{MJ}$ is appreciative to Sylvie and Philippe Villeneuve.

\section{Authors' Contributions}

Conceived the transformation between the clinical scale and the scoring for statistical analysis: AJ (senior project researcher, scientific bachelor degree). Initiators of reflection for the transformation of the Posturodynamic score: MJ and PD. Wrote the manuscript MTG MJ (MTG main writer). AJ MTG JB PD MJ participated interpretation and revising the paper. All the authors read and approved the final manuscript.

\section{Competing Interests}

The authors declare that they have no competing interests.

\section{References}

[1] Janin, M. (2012) Correlation between Clinical and Kinetic Testing in Sport Podiatry. Ter. Man, 12, 7-11.

[2] Villeneuve, P. (1995) L’épreuve posturodynamique. In: Gagey, P.-M. and Weber, B., Eds., Entrées du Système 
Postural Fin, Masson, Paris, 51-56.

[3] Weber, B., Villeneuve, P. and Villeneuve-Parpay, S. (2002) Epreuve posturodynamique chez le sujet sain. Comparaison de sa cotation qualitative par plusieurs examinateurs. In: Lacour, M., Ed., Contrôle postural pathologie et traitement, innovations et rééducation, Solal, Coll. Posture et Equilibre, Marseille, 21-23.

[4] Xia, Q., Wang, S., Passias, P.G., et al. (2009) In Vivo Range of Motion of the Lumbar Spinous Processes. European Spine Journal, 18, 1355-1362. http://dx.doi.org/10.1007/s00586-009-1068-8

[5] Roussel, N.A., Nijs, J., Truijen, S., et al. (2007) Low Back Pain: Clinimetric Properties of the Trendelenburg Test, Active Strait Leg Raise Test, and Breathing during Active Straight Leg Raising. Journal of Manipulative and Physiological Therapeutics, 30, 270-278. http://dx.doi.org/10.1016/j.jmpt.2007.03.001

[6] Johan, T. and Eva, H. (2009) Inter-Rater Reliability of Three Standardized Functional Tests in Patients with Low Back Pain. BMC Musculoskeletal Disorders, 2, 10-58.

[7] Youdas, J.W., Madson, T.J. and Hollman, J.H. (2010) Usefulness of the Trendelenburg Test for Identification of Patients with Hip Joint Osteoarthritis. Physiotherapy Theory and Practice, 26, 184-194. http://dx.doi.org/10.3109/09593980902750857

[8] Youdas, J.W., Mraz, S.T., Norstad, B.J., et al. (2007) Determining Meaningful Changes in Pelvic-on-Femoral Position during the Trendelenburgtest. Journal of Sport Rehabilitation, 16, 326-335.

[9] Hardcastle, P. and Nade, S. (1985) The Significance of the Trendelenburg Test. Journal of Bone and Joint Surgery, 67, 741-746.

[10] De Kegel, A., Dhooge, I., Cambier, D., et al. (2011) Test-Retest Reliability of the Assessment of Postural Stability in Typically Developing Children and in Hearing Impaired Children. Gait Posture, 33, 679-685. http://dx.doi.org/10.1016/j.gaitpost.2011.02.024

[11] McKeon, P.O. and Hertel, J. (2008) Systematic Review of Postural Control and Lateral Ankle Instability, Part I: Can Deficits Be Detected with Instrumented Testing. Journal of Athletic Training, 43, 293-304. http://dx.doi.org/10.4085/1062-6050-43.3.293

[12] Enoch, F., Kjaer, P., Elkjaer, A., et al. (2011) Inter-Examiner Reproducibility of Tests for Lumbar Motor Control. BMC Musculoskeletal Disorders, 25, 112-114. http://dx.doi.org/10.1186/1471-2474-12-114

[13] Keenan, A.M., Redmond, A.C., Horton, M., et al. (2007) The Foot Posture Index: Rasch Analysis of a Novel, Foot Specific Outcome Measure. Archives of Physical Medicine and Rehabilitation, 88, 88-93. http://dx.doi.org/10.1016/j.apmr.2006.10.005

[14] Granger, C.V., Carlin, M., Linacre, J.M., et al. (2010) Rasch-Derived Latent Trait Measurement of Outcomes: Insightful Use Leads to Precision Case Management and Evidence-Based Practices in Functional Healthcare. Journal of Applied Measurement, 11, 230-243.

[15] Linacre, J.M. (2004) Rasch Model Estimation: Further Topics. Journal of Applied Measurement, 5, 95-110. 